Proceedings of the 1998 IEEE/RSJ

Intl. Conference on Intelligent Robots and Systems

Victoria, B.C., Canada • October 1998

\title{
Solder Joints Inspection Using Neural Network and Fuzzy Rule-Based Classification
}

\author{
Kuk Won Ko and Hyung Suck Cho \\ Department of Mechanical Engineering \\ Korea Advanced Institute of Science \& Technology \\ 373-1 Kusong-Dong, Yusung-Gu, Taejon, \\ 305-701,Korea \\ e-mail : kkw@lca.kaist.ac.kr \\ hscho@lca.kaist.ac.kr
}

\author{
Jong Hyung Kim and Jae Son Kim \\ Vision Research Gr., FA research Institute, \\ Production Engineering Center, \\ Samsung Electronics Co. LTD, \\ 416, Maetan 3 Dong, Suwon, Kyungki-Do, \\ 441-742, Korea \\ e-mail : jhkim@srtf.sec.samsung.co.kr \\ jskim@srtf.sec.samsung.co.kr
}

\begin{abstract}
In this paper, we described an approach to automation of visual inspection of solder joint defects of SMC(surface mounted components) on PCBs (Printed Circuit Board) by using neural network and fuzzy rule-based classification method. Inherently, surface of the solder joints is curved, tiny and specular reflective; it induces a difficulty of taking good image of the solder joints. And the shape of the solder joints tends to greatly vary with soldering condition and the shapes are not identical each other, even though solder joints belong to a set of the same soldering quality, This problem makes it difficult to classify the solder joints according to their qualities. Neural network and fuzzy rulebased classification method is proposed to efficiently make human-like classification criteria of the solder joint shapes. The performance of the proposed approach is tested on numerous samples of commercial computer PCB board and compared with the human inspector performance.
\end{abstract}

\section{Introduction}

Solder joint inspection has been a critical issue for quality control in PCB assembly process. The surface of solder joint on surface mounted components(SMC) is curved, tiny and specular reflective and tends to greatly vary with soldering conditions. In the aspect of classification, even though solder joints belong to a set of the same soldering quality, the shapes are not identical each other, but vary to a certain degree. Additionally, classification criteria for solder joint inspection by human's skillful experience are difficult to describe the quantitatively so as to be programmed.

The previous researchers have developed many inspection algorithms by using statistical, rule-based expert system, neural network approach [1-9] in the area of solder joint inspection to overcome the above mentioned problems. Those methods can not give good classification performance perfectly as a human skillful ability.

In this study, a neural network and fuzzy rule-based classification approach for solder joint inspection is proposed to reflect the human experience and preknowledge on making a good criteria to classify the complex solder joints.

The architecture of the proposed neural network and fuzzy rule-based classification scheme has hierarchically two modules; neural network clustering algorithm[5,10] and fuzzy rule-based classification method[11,12]. The neural network clustering module consists of three learning vector quantization (LVQ) clustering modules. Each LVQ module is to classify solder joint color patterns by a threecolor circular illumination system according to similarity measure between input data and feature vectors of each class. Its classification rule is a simple rule, "called minimum distance criterion". However, this classification rule can not always give a good solution for the data located near the class boundaries. It is also difficult to reflect expert's knowledge on classification criteria. To overcome this problem, fuzzy rule-based classification criteria is combined into the LVQ algorithms.

The classification of solder joint is executed by fuzzy rule-based classification method with pre-defined fuzzy rules by considering each fuzzy fitness of each output of the LVQ modules. The advantages of this approach in the solder joint inspection are that the expert knowledge is reflected on classification criteria for complex solder joints, and therefore accurate classification result closer to human inspector's.

To evaluate the classification performance of the proposed algorithm, a series of experiments is carried out.

\section{Solder Joint Images}

\subsection{Illumination system}

A three color tiered illumination system is shown in Fig. 
1, which consists of three colored circular lamps (Red, Green, Blue), a CCD camera, a color image processing board and IBM compatible PC with a display monitor. The lamps are coaxially tiered in the sequence of green, red and blue upward from the bottom of inspection surface. The three-color lamps illuminate solder joint surface with different incident angles: the blue lamp to $20^{\circ}$, the red lamp to $40^{\circ}$, the green lamp to $70^{\circ}$.

The CCD color camera system has 1:7 zoom lens giving a field of view of $10 \mathrm{~mm}(\mathrm{H}) \times 10 \mathrm{~mm}(\mathrm{~V})$ on PCB to acquire ten or eleven solder joints in an image.

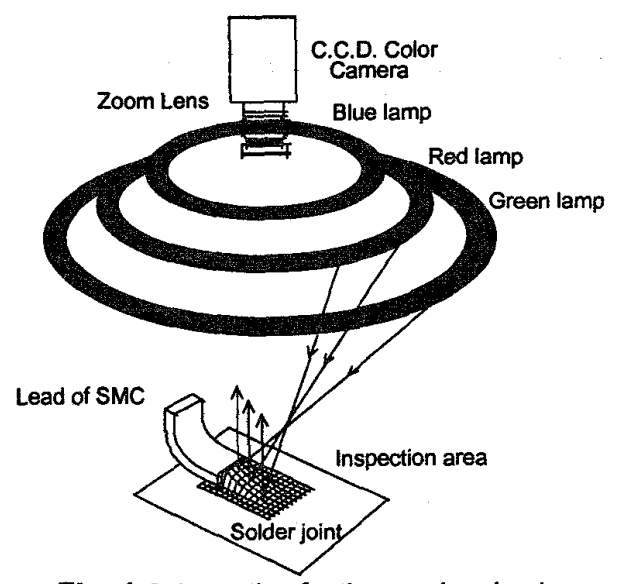

Fig. 1 Schematic of a three color circular illumination system.

\subsection{Color image of solder joints}

The interesting target that is concerned here is the solder joint of SMC. Fig. 2 shows an image of solder joints on the PCB under the illumination system. The color patterns on the specular surface such as leads and solder joints are generated according to surface slope.

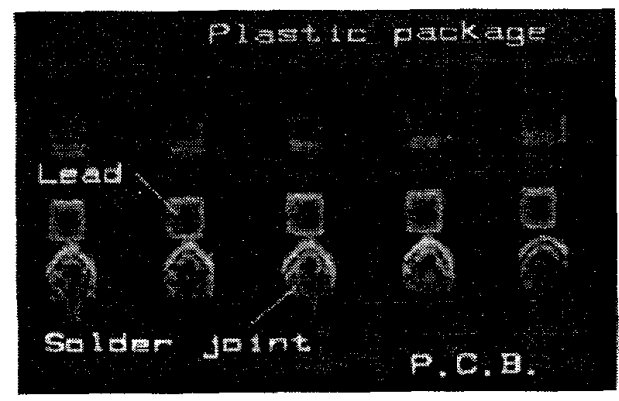

Fig. 2 A typical image of PCB under the illumination system.

In this study, the qualities of solder joint are divided into five classes; insufficient soldering(I), insufficient but acceptable soldering(IA), acceptable soldering(A), excess but acceptable soldering(EA), and excess soldering(E).
Synthetic 3-D joint shapes and typical corresponding schematic color patterns according to the classes of soldering qualities are shown in Fig. 3:

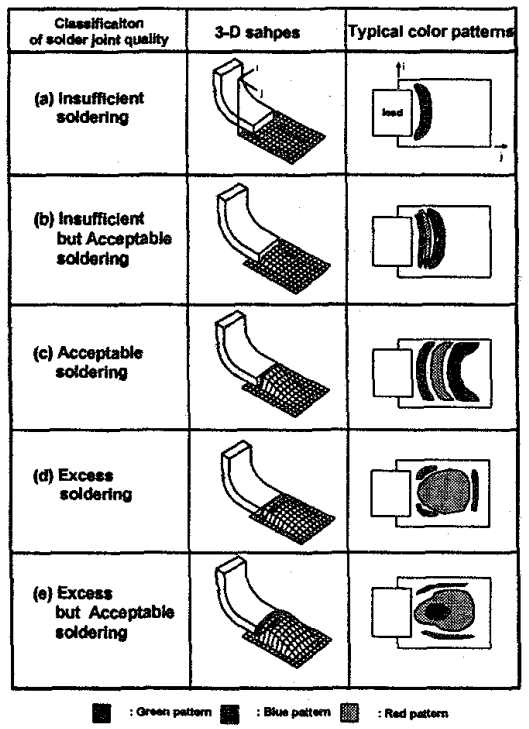

Fig. 3 Solder joint shapes and the corresponding color patterns

1) The insufficient soldering has most flat surface or very small fillet. Therefore, the blue pattern dominantly appears in the solder joint image(Fig. 3(a)).

2) As amount of solder increases, joint surface begins to form fillet joining the lead and the solder pad. Since the insufficient but acceptable soldering has a small fillet, the red and green patterns begin to appear though their sizes are still small (Fig. 3(b)).

(3) The acceptable solder joint has larger fillet than those of the previous two soldering classes and looks like a concave solder fillet. Thus, the green, red, and blue patterns appear sequentially from the lead. This indicates that the surface near to the lead is inclined steeply and the joint surface connected with solder pad is nearly flat (Fig. 3(c)).

(4) The excess but acceptable soldering usually has an inclined flat surface, such that the red pattern appears dominantly in the window image (Fig. 3(d))

(5) The excess solder joint has a convex solder fillet so that blue pattern is enclosed by the red and green color patterns. The sequence of color patterns from the lead is different from those of the other soldering classes (Fig. 3(e)).

The visual cues presented in the above are the color patterns on the solder joint image and can be somewhat distinct. These color patterns will be used to distinguish the quality of the solder joint. 


\section{Neural Network and Fuzzy Rule- based Classifier}

In this section, we describe the proposed neural network and fuzzy rule-based classification scheme. Its architecture of the proposed classification scheme consists of two modules; three LVQ clustering module and fuzzy rule based classification module as shown in Fig 4 . In the neural network clustering module, which is based on the LVQ algorithm, generates the prototype patterns, and evaluate fitness in each classes by using the similarity between an input pattern and prototype patterns. In the fuzzy classification module, the classification is performed by considering each fuzzified output values from the clustering module with pre-defined rules in which expert knowledge can be imbedded.

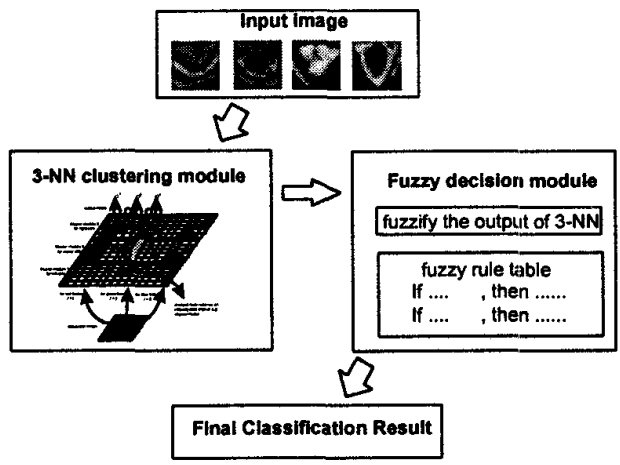

Fig. 4 The architecture of the proposed classification scheme

\subsection{Neural network clustering modules}

The proposed NN clustering module is based on LVQ algorithm, which is well known as $\mathrm{NN}$ self-clustering classifier[5,10]. The structure of NN clustering module is shown in Fig. 5.

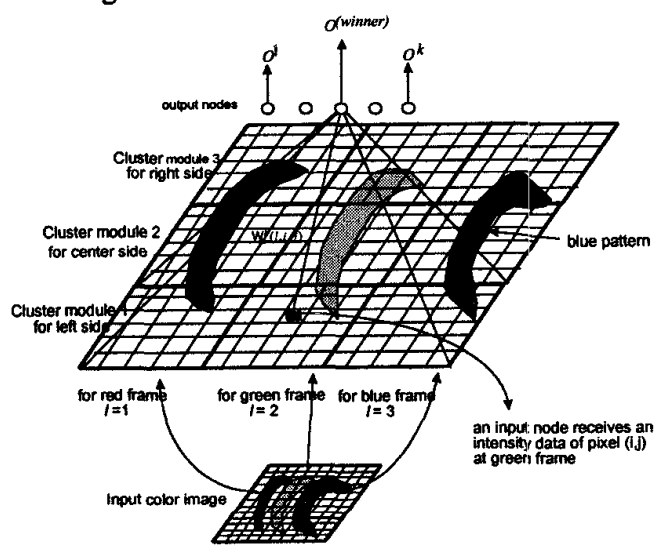

Fig.5 The structure of $\mathrm{NN}$ clustering module
The input data of the module is the color intensities of each pattern from pixel to pixel shown in the form of threecolor patterns obtained under the three-color circular illumination. The dimension of input nodes is the same as that of the input images. Output nodes are fully connected to all input nodes by the weights. In the self-clustering module, the input value to the output node is a similarity measure between an input image and the weights, which are connected to the output nodes. The output nodes compete with each other and only one neuron, which has the weights closest to the current input data, is selected as a winner by the well-known competitive learning rule. The weight of the winner output node is to be updated towards resembling the members in its own cluster.

The training producer of LVQ clustering module are described as follows:

First, all the NN parameters such as the number of output node, dynamic learning rate are defined and the weights is randomly assigned between 0 and 255 . Select a sample randomly and apply this sample to self-clustering module, and then determine the winner output, $W_{c}^{\text {win }}$ by

$$
\begin{gathered}
\left\|\mathbf{W}_{c}^{\text {win }}-\mathbf{I}_{\mathrm{c}}\right\|<\left\|\mathbf{W}_{c}^{k}-\mathbf{I}_{\mathrm{c}}\right\|, \text { forall } k, \mathrm{k} \neq \mathrm{c} \text { or } \\
\sum_{\mathrm{i}}^{\mathrm{m}} \sum_{j}^{n} \sum_{l}^{3}\left|W_{c}^{\text {win }}(\mathrm{l}, \mathrm{i}, \mathrm{j})-I_{\mathrm{c}}(l, i, j)\right|<\sum_{\mathrm{i}}^{\mathrm{m}} \sum_{j}^{n} \sum_{l}^{3}\left|W_{c}^{k}(1, \mathbf{i}, \mathrm{j})-I_{\mathrm{c}}(l, i, j)\right|
\end{gathered}
$$

where subscript $c$ indicates the class of the input data, superscript $k$ indicates the label of output node, $I_{\mathrm{c}}\left(l, i_{2}\right)$ means an intensity value at the $(i, j)$ pixel in the $l$ color frame ( $1=$ red, $2=$ green, $3=$ blue), $(i, j)$ indicates a pixel position, and $(n, m)$ indicates the size of a window image. Next, update the weights of the winning node as follows:

$$
W_{c}^{\text {win }}(t+1)=W_{c}^{\text {win }}(t)+\eta(t) \cdot\left\{I_{c}-W_{c}^{\text {win }}(t)\right\}
$$

where $t$ is the number of iterations for learning, and the $\eta(t)$ is dynamic learning rate that decreases as $t$ increases. Repeat the above learning procedure until all training input images are assigned, and the predefined learning step reaches. After training the procedure, adjust the weights more accurately in the following manner: If the input data are misclassified into a different cluster, update the prototype image of the cluster again. Take for example; consider the input data $\mathrm{I}_{0}$ belonging to the $p$-th class. If it is misclassified into the cluster labeled as the $c$-th class, then update the weights of the cluster as follows:

$$
W_{c}^{\text {win }}(t+1)=W_{c}^{\text {win }}(t)+\eta(t) \cdot\left\{I_{p}-W_{c}^{\text {win }}(t)\right\}
$$

The above equation indicates that it adjusts the decision surface between two classes by moving the winner prototype image in the opposite direction, away from the sample data. After training procedure, the trained weights of each output node remember the averaged color 
intensities of its own family.

\subsection{Fuzzy rule-based classification}

Fuzzy set theory provides a conventional tool for roughly representing the concept of a process using natural language and reasoning a correct decision from a collection of linguistic rules[11,12]. The basic concept of fuzzy decision for classification of solder joint is to evaluate the classification rule using the similarity measure between an input fuzzy membership from the output of NN clustering module and classification fuzzy rule criteria. The calculation of classification result are composed of following method:

First, the classification result of $\mathrm{NN}$ clustering module is evaluated and the output values of $\mathrm{NN}$ clustering module are converted to fuzzy variables. Second, the classification rules using the compositional rule of inference is evaluated, and finally the best classification from the result of evaluated decision value is found. The typical classification rule is defined in the following linguistic form.

IF the shape in the right column window

AND the shape in the center column window

AND the shape in the left column window

is $(I, I A, A, E A, E)$

THEN the classification result

is $(I, I A, A, E A, E)$ is $(I, L A, A, E A, E)$

where I,IA,A,EA,E is the insufficient soldering, the insufficient but the acceptable soldering, the acceptable soldering, the excessive but acceptable soldering, the excessive soldering, respectively. In this rule, the premise of fuzzy classification rule estimates the shape of solder joint from the output of fuzzy clustering module. The consequence gives the classification on the quality of solder joint.

\section{Experimental Results and Discussions}

To evaluate the performance of the proposed method, a series of experiments was performed for various solder joints. First the learning performance of the proposed method was evaluated. Secondly, the classification performance is evaluated. Finally, comparison studies are performed with the results by a human inspector and LVQ(learning vector quantization) classifier

\subsection{Sample data}

The sample images of the solder joints for training are collected from five commercially manufactured PCBs. The 256 samples selected randomly are used for training, and the 96 samples are used for testing. All samples are divided by an expert inspector into five groups according to their qualities. Each sample image is a window sub-image fitted to a solder joint with a size of $55 \times 45$ pixels as shown in Fig. (6).

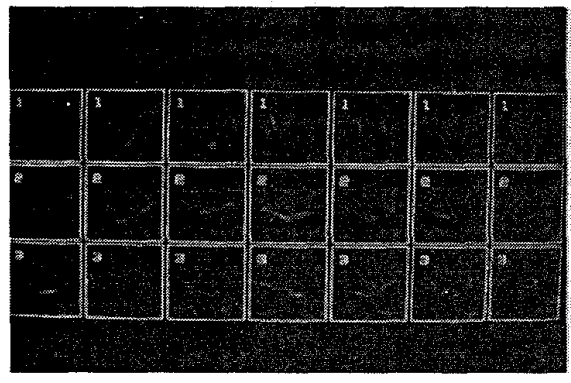

Fig. 6 Solder joint image for experiments

\subsection{Training the clustering module}

The NN clustering module consists of three sub-clustering modules: one of them for shape in the center of solder joint, the rest of them for the classification of shapes in the both sides of solder joint. The shapes in both sides of the solder joint are different from those in the middle of solder joint. The windows on a solder joint are divided into three columns as shown in Fig. 7.

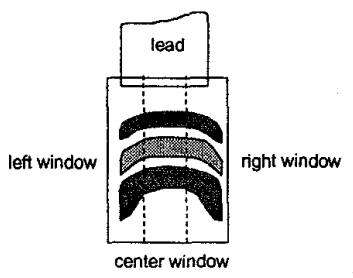

Fig. 7 The windows on solder joint

Input: The input sets of each LVQ clustering module are the pixels of the color patterns in three sub-images at predefined window. The input sets are formed of a two dimensional array of $15 \times 165$ pixels for both sides, $15 \times$ 165 pixels for center. The $k$-th clustering center is defined by

$$
W^{k}=\left\{w^{k}(l, i, j) \mid l=1,2,3, i=1,2, \cdots 15, j=1,2, \cdots, 165\right\}(4)
$$

The number of the output node for each clustering module are assigned to 10 for side shape, and 8 for center shape considering the results of the previous studies[5,10]. The initial dynamic learning rate is set to 0.25 . Fig. 8 shows the prototypes(weights) of each module after training.

Labeling: after training data sample, the class is labeled by an expert The class which represents the insufficient quality scores 1 , the class for excess soldering 10 for the side classification, from 1 to 8 for center classification, 
according to amount of solder. The number of Fig. 8 below class is labeled class, which is used for fuzzification.

\begin{tabular}{|c|ccccccccccc|}
\hline Class & & & & & IA & & \multicolumn{1}{c}{ A } & & EA & E \\
& 1 & 2 & 3 & 4 & 5 & 6 & 7 & 8 & 9 & 10 \\
\hline Side & & & & & & & & & & & \\
Column & & & & & & & & & & \\
\hline
\end{tabular}

(a) Prototypical image for both side column window

\begin{tabular}{|c|c|cccccccc|}
\hline Class & & & I & & IA & A & EA & A \\
& & 1 & 2 & 3 & 4 & 5 & 6 & 7 & 8 \\
\hline Center & & & & & & & & \\
Column & & & & & & & & & \\
\hline
\end{tabular}

(b) Prototypical image for the center column window

Fig. 8 Trained Prototypical image

\subsection{Design of fuzzy classification rule}

The fuzzy classification rule is designed for accurate classification of solder joint with expert knowledge. The classification criteria is based on the knowledge of inspector. Table 1 shows one example of fuzzy rules for the classification of insufficient soldering.

Table 1. Fuzzy rule for insufficient soldering

\begin{tabular}{|c|c|c|c|c|}
\hline & \multicolumn{3}{|c|}{ Premise } & Consequence \\
\cline { 2 - 4 } & $\mathrm{R}$ & $\mathrm{C}$ & $\mathrm{L}$ & \\
\hline $\mathrm{R} 1$ & $I$ & $I$ & $I$ & $I$ \\
\hline $\mathrm{R} 2$ & $I$ & $I A$ & $I$ & $I$ \\
\hline $\mathrm{R} 3$ & $I$ & $I$ & $I A$ & $I$ \\
\hline $\mathrm{R} 4$ & $I \boldsymbol{A}$ & $\boldsymbol{I}$ & $\boldsymbol{I}$ & $\boldsymbol{I}$ \\
\hline $\mathrm{R} 5$ & $\boldsymbol{I} \boldsymbol{A}$ & $\boldsymbol{I}$ & $\boldsymbol{I} \boldsymbol{A}$ & $\boldsymbol{I}$ \\
\hline
\end{tabular}

$\mathrm{R}$ : right module C: center module, L: left module

Fig. 9 shows the fuzzy membership function for each subclass. Fig. 9(a) shows the membership function for both sides of solder joint, while Fig. 9(b) membership function for center of solder joint. The shape of membership function is initially defined by inspector's knowledge and is modified by considering classification result. For input variables to fuzzy classification module, the outputs of each NN clustering modules are converted to fitness values defined as equation (5). This fineness value means the degree of similarity of two best similar classes for a current input image.

$$
\begin{aligned}
O(x) & =f\left(c_{1}, c_{2}, d^{c 1}(x), d^{c 2}(x)\right) \\
& = \begin{cases}c_{1} \times \frac{d_{i}^{c 1}(x)}{d^{c 1}(x)+d^{c 2}(x)}, & \text { if } c_{1} \leq c_{2} \\
c_{2} \times \frac{d^{c 2}(x)}{d^{c 1}(x)+d^{c 2}(x)}, & \text { otherwise }\end{cases}
\end{aligned}
$$

where $x$ is the input to neural network clustering module, $d^{c_{1}}(x)$ is the maximum fitness value, $d^{c_{2}}(x)$ is the second largest fitness value and $c_{1}, c_{2}$ are the labeling value of the best and second best similarity class.

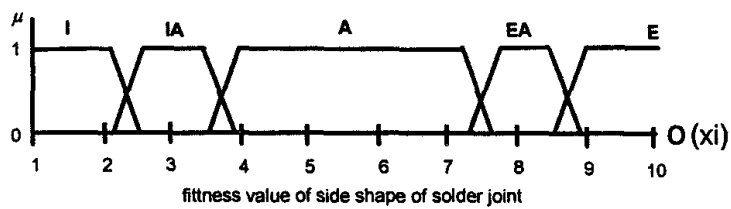

(a) Membership function for the side shapes of solder joint

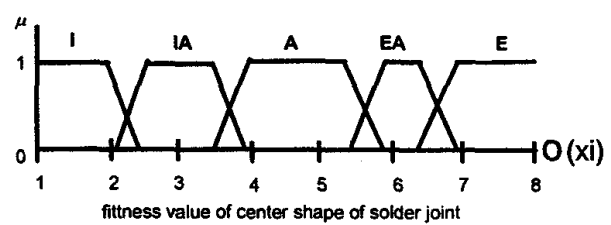

(b) Membership function for the center shapes of solder joint

Fig. 9 Fuzzy membership functions

For the input sample as shown in Fig. 10 is classified by 3LVQ modules. The best fitness class by LVQ is $2,2,2$ for right, center and left, and each result is calculated by equation (5). The each result value by equ. (5) is $2.1,2.2$, 2,18 respectively. These values are fuzzified by predefined fuzzy membership value as shown in fig. (8). The fuzzified result is judged by predefined rule as shown in table 1 . In this rule, the quality of solder joint is to be judged by considering the shapes of three windows on solder joint images. The classification result is shown in table 2.

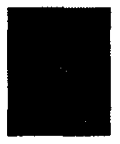

Fig. $10 \mathrm{~A}$ test image for fuzzy rule-classification

Table 2. Classification result of insufficient soldering

\begin{tabular}{|c|c|c|l|c|}
\hline \multicolumn{3}{|c|}{ Premise } & fitness & cons. \\
\hline $\mathrm{R}$ & $\mathrm{C}$ & $\mathrm{L}$ & & \\
\hline$I: 0.7$ & $I: 0.8$ & $I: 0.6$ & $I:(0.7+0.8+0.6) / 3=0.76$ & $I$ \\
\hline IA: 0.3 & $I A: 0.2$ & IA $: 0.4$ & IA $:(0.3+0.2+0.4) / 3=0.3$ & \\
\hline
\end{tabular}

\subsection{Classification performance}

To test the classification performance of the proposed algorithm, the classification result is compared to that of the expert inspector. Table 3 shows the classification results for the training data. Result appears to be satisfactory because the training data of five quality classes are accurately classified. 
Table 3. Classification result for training samples

\begin{tabular}{|c|c|ccccc|c|}
\hline Q & NS & I & IA & A & EA & E & NC \\
\hline I & 48 & 48 & & & & & 0 \\
IA & 23 & & 23 & & & & 0 \\
A & 116 & & & 116 & & & 0 \\
EA & 28 & & & & 28 & & 0 \\
E & 41 & \multicolumn{7}{c|}{ Total success rate $: 1-0 / 256=1.00(100 \%)$} \\
\hline \multicolumn{7}{|c|}{}
\end{tabular}

Q: quality, NS: no. of samples, NC: no. of confusion

Table 4 shows the classification results for the test data that are not used for the training. For class (I), (A), (E), all training data are correctly classified. For class (IA), (EA), 2 samples are misclassified into class (I) and (A). For these two classes, the boundaries between these classes are not distinct. An expert inspector can not exactly classify either the difference among the solder joint shape for the class (EA), (IA).

Table 4. Classification result for the test samples

\begin{tabular}{|c|c|ccccc|c|}
\hline Q & NS & I & IA & A & EA & E & NC \\
\hline I & 18 & 18 & & & & & 0 \\
IA & 13 & 1 & 21 & 1 & & & 2 \\
A & 30 & & & 30 & & & 0 \\
EA & 14 & & & & 12 & 2 & 2 \\
E & 21 & \multicolumn{7}{c|}{ Total success rate : $1-4 / 96=0.9583(95.83 \%)$} \\
\hline \multicolumn{7}{|c|}{}
\end{tabular}

Q: quality, NS: no. of samples, NC: no. of confusion

\subsection{Comparison study}

Table 5 shows the clustering results by using a conventional LVQ self-clustering algorithm. In this experiment, the number of initial output nodes are set to 10 , and the weight is dynamically decreased to 0 with time. The training input data are classified into ten prototype images. As can be seen form the table 5, LVQ classifier used in this case can not accurately classify the training data, although the total number of class is larger than the proposed method.

Table 5. Classification result based on LVQ algorithm

\begin{tabular}{|c|c|c|c|c|c|c|c|c|c|c|}
\hline \multirow[t]{2}{*}{$\mathrm{Q}$} & \multirow[t]{2}{*}{ NS } & I & IA & \multicolumn{2}{|l|}{ A } & \multicolumn{2}{|c|}{ EA } & \multicolumn{2}{|l|}{$E$} & \multirow[t]{2}{*}{$\mathrm{NC}$} \\
\hline & & 12 & 3 & 45 & 6 & 7 & 8 & 9 & 10 & \\
\hline I & 49 & 2918 & 2 & & & & & & & 2 \\
\hline IA & 21 & 6 & 13 & 2 & & & & & & 8 \\
\hline A & 115 & & 4 & 25434 & & 2 & & & & 6 \\
\hline EA & 27 & & & & 4 & 6 & 10 & 7 & & 11 \\
\hline E & 44 & & & & & 1 & 4 & 23 & 16 & 5 \\
\hline
\end{tabular}

Q: quality, NS: no. of samples, NC: no. of confusion

\section{Conclusions}

A classification method using neural network and fuzzy rule-based classification method has been implemented for the solder joint inspection. A series of experiments was performed for various solder joints of commercially manufactured PCBs. Experimental results show the practical usefulness of the proposed method for solder joint inspection. The advantage of this approach in the solder joint inspection is that expert knowledge can be reflected on classification criteria for complex solder joints. As a result, the proposed method was found to be more accurate than conventional unsupervised clustering algorithms.

\section{References}

1. Bartlett S.L, Besl. P.J, Jain R, Mukherjee. D and Skifstad K.D, "Automatic Solder Joint Inspection", IEEE Trans, PAMI, Vol. 10, No. 1 pp. 31-43, 1988.

2. Ikeuchhi, K., "Determining Surface Orientations of specular Surfaces by using the Photometric Stereo Method", IEEE Trans PAMI, Vol. 3, No. 6, pp 661-669, 1981.

3. Nayar S. K., Sandreson. A.C., Weiss L. E. and Simson D.D, "Specular Surface Inspection using Structured highlight and Gaussian Images", IEEE Trans, Robotics and Automation, Vol. 6, No. 2, pp. 108-218, 1990.

4. Capson D.W. and Eng S.K., "A Tiered Color Illumination Approach for Machine Inspection of Solder Joint ", IEEE Trans. PAMI, Vol. 10, No. 3, pp 387-393, 1988.

5. J. K. Kim and H. S. Cho, "Neural Network-based Inspection of Solder Joints using a Circular Illumination", Image and Vision computing, Vol. 13, pp. 479-490. 1995.

6. Park J.S. and Tou J. T., "A Solder Joint Inspection system for automated printed circuit board manufacturing", Proceeding of Int. Conf. on Robotics and automation, pp. 1290-1295,1990

7. K.W.Ko, J.H.Kim, and H.S.Cho, "Solder Joints Inspection using a Circular Illumination \& Fuzzy Pattern matching", ICARCV'96, pp1044-1048, 1996.

8. H.S.Cho, J.S.Kim, J.H.Kim, "Development of Visual Inspection Machine for Solder Joint", KAIST LCA Tech. Report, 1993.

9. H.S.Cho, K.W. Ko, I.S. Joung, "Development of Advanced Solder Joint Inspection System for SMC", KAIST LCA Tech. Report, 1996.

10. Jacek M. Zurada, "Introduction to Artificial Neural Systems", West Publishing company, 1992.

11. J.C. Bezdeck, "Pattern Recognition with Fuzzy Objective Function Algorithm", Plenum Press, 1991.

12. H.J. Zimmermann, "Fuzzy Set Theory and Its Applications", Kluwer-Nijhoff Publishing. 1992. 